\title{
Emergence of Arthropod Transmitted infections in Kennel Dogs
}

\author{
Ravindra K Jadhav, Rashmi Rekha Kumari ${ }^{1}$, Javed Jameel A, Pankaj Kumar* \\ Division of Medicine, 1. Division of Pharmacology \\ Indian Veterinary Research Institute, I zatnagar - 243122, UP, India \\ * Corresponding author email: pankajvet@gmail.com \\ Received: 13-06-2011, Accepted: 17-06-2011, Published Online: 22-09-2011 \\ doi: $10.5455 /$ vetworld.2011.522-528
}

\begin{abstract}
Changing scenario of climate resulting from global warming and adversity of nature has also resulted in emergence and re-emergence of diseases transmitted by arthropods. Increasing trends of population growth of dogs has increased the chance of disease transmission due to readily available susceptible host. Babesiosis and Hepatozoonosis and Ehrlichiosis are the main arthropod borne diseases of dogs prevalent in India. The present article explains the importance of these arthropod transmitted infections in kennel dogs, research progress and reason for their emergence in the present scenario.
\end{abstract}

Key words: Emergence, Arthropod, Babesiosis, Hepatozoonosis, Ehrlichiosis, Kennel Dogs

\section{I ntroduction}

Climatic conditions in India are quite favorable for the growth and multiplication of arthropods which act as vector for many diseases of animals including canine species. Changing scenario of climate resulting from global warming and adversity of nature has also resulted in emergence and re-emergence of diseases transmitted by arthropods. The process of disease transmission is augmented by the large population of stray dogs in our country which acts as a host for constant maintaining the transmission cycles of vector borne diseases like hemoprotozooa (Azaziah et al., 2010). Above all, the changing social structure, urbanization and industrialization, people are increasingly keeping dogs as pets for security as well as companion animal. Security concern resulting from terrorist outfits and insecurity at border areas of our country has also forced security forces (military, paramilitary and police) to increase dog squads and strength of dogs in kennels for multi-purpose use. Thus increasing population of dogs has increased the chance of disease transmission due to readily available susceptible host. Babesiosis and Hepatozoonosis and Ehrlichiosis are the main arthropod borne diseases of dogs prevalent in India. But till date our knowledge regarding the epidemiology, diagnostics, treatment and management of these arthropod borne infections is quite limited. So these infections poses a great challenge on veterinarians to improve knowledge as well as provide evidences regarding these infection by use of highly sensitive diagnostic tools like serological tests, molecular tools like PCR etc. in order to undertake therapeutic and control measures.

Studies of canine babesiosis in India are limited to epidemiological studies with prevalence of infection ranging from $0.1-22 \%$ in different parts of India on the morphological criteria by microscopic examination (Sundar et al., 2004; Chaudhari, 2006). Most of the studies regarding babesia in India are lacking information at level of species and genotype harbored by infected dogs. The pathogenicity of babesia is also known to differ in different parts of India which might be due to strain and species variation (Azaziah, et al., 2010).

Very sparse reports are available regarding the prevalence of canine Hepatozoonosis in India. It has been reported in Mumbai in dogs with characteristic clinical signs of anemia, thrombocytopenia, hepatitis, elevated blood urea nitrogen and hyperglobulinemia (Pawar and Gatne, 2005). Subclinical hepatozoon infections with prevalence ranging from 3-9\% have been reported (Gupta et al., 1994; Sharma et al., 1997). There are very few studies regarding the prevalence of Ehrlichiosis in dogs in India. Conventional diagnostics of blood smear examination for parasite morphology has shown the prevalence ranging from $0.35 \%$ in Punjab to $18.9 \%$ in Nagpur (Juyal et al., 1994; Samaradni et al., 2003). Lakshmanan et al., (2007) used nested PCR for detection of Ehrlichia from blood of dogs and found $50 \%$ dogs positive by 
nested PCR for E. canis infection compared to only $19 \%$ by blood smear examination.

\section{Canine Babesiosis}

Canine babesiosis is an important tick borne piroplasm infection caused by intraerthrocytic parasites of the genus Babesia (Taboada and Lobetti, 2006). It has worldwide prevalence in dogs and is characterized by erythrocyte destruction, anemia, pigmenturia, thrombocytopenia, $\gamma$-hyperglobulinemia, and waxing and waning of fever, pallor, jaundice, extravascular haemolysis, hypoxic injury, splenomegaly and systemic inflammation (Boozer and Macintire, 2003; Irwin, 2009).

Based on the morphological characteristics and areas of prevalence canine babesia are categorized in to large and small babesia organisms. Babesia canis is a large $(2.5-5 \mu \mathrm{m})$, piriform shaped organism generally observed as single or paired organisms in the infected erythrocytes. Trinomial classification has been proposed for B. canis based on cross-immunity, serological testing, vector specificity and molecular phylogeny (Zahler et al., 1998; Carret et al., 1999; Gallego, 2008). First one is, B. canis vogeli, least pathogenic among three, reported in tropical and subtropical regions and transmitted by Rhipicephalus sanguineus. Second, B. canis canis with intermediate pathogenicity and detected in Europe and parts of Asia transmitted by Dermacentor ticks. Third species, $B$. canis rossi is most pathogenic among three, which is prevalent in South Africa and transmitted by Haemophysalis leachi ticks. With advent of molecular methods, newer species that infect dogs have been identified like, B. conradae (Kjemtrup and Conrad, 2006), B. microti like (Camacho-Garcia, 2006) and unnamed large Babesia spp. (Birkenheuer et al., 2004).

Small babesia constitutes B. gibsoni which is a small (1-3.2 $\mu \mathrm{m})$, pleomorphic organism usually observed singly within erythrocyte. It is distributed over Northern America, Southern Asia, Australia, Europe and USA and transmitted by several species of Ixodid ticks. In addition to role of Ixodid ticks in transmission of B. gibsoni, non-vector transmission by exchange of blood during fighting and biting has been reported in USA. Pit bull terrier dogs have more prevalence of $\mathrm{B}$. gibsoni infections that might correlates to frequent use of these dogs for fighting (Yeagley et al., 2009).

Babesia parasite multiplies by binary fission in erythrocytes. Infected erythrocytes incorporates parasite antigen $(\mathrm{Ag})$ into their surface which induces production of antibodies (Abs). Opsonization of erythrocytes with Abs leads to removal of infected erythrocytes by mononuclear phagocyte system (MPS). The major mechanisms of pathogenesis include intravascular and extravascular hemolysis culminating in progressive anemia, hemoglobinemia, hemoglobinuria, bilirubinuria and icterus. Inadequate oxygenation of tissues due to anemia further causes hypoxic tissue injury leading to disseminated intravascular coagulopathy (DIC), and irreversible shock leading to death. Two main syndromes are produced by babesia which includes hemolytic anemia and multiple organ dysfunction syndrome (MODS) due to systemic inflammatory response syndrome.

Clinical presentation of babesia infection varies from hyperacute, acute to chronic or subclinical. Acute syndrome is characterized by fever, anorexia, lethargy, acute hemolytic anemia, thrombocytopenia, swelling of lymphnodes, spelnomegaly. Signs like vomiting; hematuria and icterus are reported in dogs with $B$. canis infection. Hyperacute syndrome is very severe and characterized by extensive tissue damage but reported very rarely. Chronic form of babesiosis is poorly characterized and might be manifested as low grade or subclinical syndrome. Two forms of babesiosis have been observed in dog's viz., uncomplicated and complicated babesiosis.

Uncomplicated form is characterized by acute haemolysis, fever, anorexia, depression, lethargy, pale mucus membranes, splenomegaly, and lymphadenomegaly. While complicated babesiosis is characterized by simultaneous pathology in various organ systems like, acute renal failure, cerebral babesiosis, coagulopathy, icterus, hepatopathy, immune mediated hemolytic anemia, acute respiratory distress syndrome, hemoconcentration, hypotension, cardiac changes, acute pancreatitis, acidbase disturbances etc. (Taboada and Lobetti, 2006).

Diagnosis is done conventionally by Wright, Giemsa or Diff-Quick staining of blood smear prepared from peripheral blood. Examination of blood smear under oil immersion lense used to identify morphology of parasite. B. canis appears as large, piriform organisms which exist either as single or in pairs in erythrocytes. Serological tests like indirect fluorescent antibody tests (IFAT), enzyme linked immunosorbent test (ELISA) have been developed, sidetracked due to limited sensitivity and specificity.

These tests are generally employed for seroepidemiological studies. Nucleic acid detection by genus specific screening of DNA extracted from 
blood is performed by Polymerase Chain Reaction (PCR). Semi nested PCR even used for definitive diagnosis and differentiation of $B$. gibsoni and all subspecies of $B$. canis with defined limit of detection which could improve diagnostic efficacy for babesia infections (Birkenheuer et al., 2003). Recently application of quantitative fluorescent resonance energy transfer (FRET)-PCR to amplify $18 \mathrm{~S}$ rRNA gene fragments of babesia spp. shown high sensitivity and specificity as well as species differentiation based on disassociation temperature of the fluorescent probes (Wang et al., 2010).

Line of treatment for canine babesiosis varies with the severity of infection and accompanying complications. Diminazene aceturate is the widely practiced drug across the world (Irwin, 2009). It is effective when given intramuscularly (IM) but clearance of parasite in not consistent. On the other hand drug exerts some toxic effects in predisposed canines. Imidocarb dipropionate is an effective therapeutic agent against $B$. canis infection in dogs. A single dose of $6-7.5 \mathrm{mg} / \mathrm{kg}$ the day following a regular dose of diminazene at $3.5 \mathrm{mg} / \mathrm{kg}$ has shown efficacy in clearing the infection. But all these drugs are not extensively studied as Diminazene aceturate. Other drugs that have been exploited for managing clinical signs for many years include Quinuronium sulphate, trypan blue, pentamidine, phenamidine and parvaquone (Irwin, 2009). Newer successful treatment modalities have been reported to have encouraging response in cure of $B$. gibsoni infection in dogs which includes, combination of clindamycin, metronidazole@15 mg/kg every 12 hours orally and doxycycline @ $5 \mathrm{mg} / \mathrm{kg}$ every 12 hours orally (Suzuki et al., 2007) and azithromycin @ $10 \mathrm{mg} / \mathrm{kg}$ every 24 hours orally with atovaquone @ $13.3 \mathrm{mg} / \mathrm{kg}$ every 8 hours orally for 10 days with good efficacy and safety (Birkenheuer et al., 2004). Clindamycin @ $25 \mathrm{mg} / \mathrm{kg}$ orally every 12 hours for 1-3 weeks can be effective if other antibabesial drugs are not available. Doxycycline has also been found effective in preventing or reducing the severity of parasitemia in dogs. In case of severe anemia and dehydration, supportive therapy in the form of blood transfusion, hematinic, fluid therapy, etc. is required.

\section{Canine Hepatozoonosis}

It is an arthropod borne hemoprotozoon infection of canines caused by Apicomplexan protozoa Hepatozoon. Infection is transmitted by various arthropod vectors like ticks, mites, mosquitoes, sandflies, tsetse fly, flea, lice and reduviid bugs. Mode of transmission of Hepatozoon infection is unique. Unlike other vector borne protozoon infections, Hepatozoon infection in canines occurs by ingestion of vector containing mature oocytes of Hepatozoon in hemocoel. Two species of Hepatozoon are prevalent among dogs across the world, viz., Hepatozoon canis and Hepatozoon americanum both of which produce unique clinical syndrome in infected dogs.

Hepatozoon canis infection (HCI) was first described in India in 1905 (Craig, 2006). HCI is unique infection in which tick vector acts as a definitive host for parasite where as dogs are intermediate hosts from which ticks acquire infection of gamonts through ingestion of infected leukocytes. HCI is prevalent among dogs in tropical, subtropical as well as temperate climates. There is no gender and breed predilection and disease is reported in dogs of all age groups. Dogs acquire infection by ingestion of ticks infected with Hepatozoon canis. Brown dog tick (Rhipicephalus sanguineus) acts as a primary vector. Transtadial transmission has been reported. Horizontal transmission through uterus from dam to offspring may also occur.

After ingestion of vector tick by dog, sporozoites are released in to intestine which invades mononuclear cells after penetration of gut wall and disseminate hematogenously or via lymph to hemolymphatic target organs. Target organs include bone marrow, spleen, lymphnodes and liver, kidneys and lung. It may culminate in development of hepatitis, glomerulonephritis, pneumonia etc. Meronts (previously called schizonts) develop in to asexually dividing merozoites in dog tissues by process of merogony. They are of two types' viz., macromierzoites or micromerozoites. Merozoites released from mature meronts invade neutrophils as well as monocytes and undergo gamontogony to produce gamonts (previously called as gametocytes). Ticks become infected by ingestion of leukocytes containing these gamonts during blood meal on dog host. Gametogenesis in gut of tick produces distinct gamets which after fertilization produce zygote. Sporogony occurs in tick hemocoel to produce oocysts containing 100 s of sporocysts containing sporozoites infective to dogs.

Immunodeficient dogs are susceptible to HCI infection. HCI infection shows variety of clinical patterns. Most common clinical presentation is low level parasitemia (less than 5\% neutrophils) which may go asymptomatic or show mild disease and is characterized by anemia, lowered platelet count. Rare but very severe disease is characterized by high 
parasitemia (approaching infection of $100 \%$ neutrophils with leukocytosis). Severe syndrome is characterized by fever, lethargy, weight loss, anemia and $\gamma$-hyperglobulinemia. In highly parasitemic dogs load of blood and tissue parasites take its toll by demanding nutrients and exerting tissue injury.

$\mathrm{HCI}$ is diagnosed on the basis of clinical pattern of syndrome and laboratory findings like leukocytosis, extreme neutrophilia (more than 10000/ $\mu$ l), anemia (normochromic, normocytic), serum biochemical changes like increased levels of serum Creatinine kinase (CK) and Alkaline phosphatase (ALP) as well as $\gamma$-hyperglobulinemia. Examination of Giemsa stained blood smears reveals presence of ellipsoidal gamonts $(11 \mathrm{x} 4 \mu \mathrm{m})$ in cytoplasm of neutrophils and occasionally in monocytes. Histopathology of tissue sections or cytology also aids in detection of meronts. Serological tests like IFAT and ELISA by employing purified gamont antigens is generally used for epidemiological studies. Generally antibodies of IgM and $\mathrm{IgG}$ are produced against parasite as early as 16 and 22 days post infection respectively, which peaks at 7-9 weeks and persists for up to 7 months (Gonen et al., 2004).

Imidocarb dipropionate is a choice drug used @ $5-6 \mathrm{mg} / \mathrm{kg}$ IM or subcutaneously (SC) every 14 days until blood smears were negative for gamonts. Doxycycline @ $10 \mathrm{mg} / \mathrm{kg} /$ day orally for 21 days with Imidocarb dipropionate is commonly used in practice to treat identified or potential co-infections (Craig, 2006). Also combination of Tetracycline hydrochloride (a $22 \mathrm{mg} / \mathrm{kg}$ every 8 hours and Imidocarb dipropionate @ $6 \mathrm{mg} / \mathrm{kg} \mathrm{SQ}$ every 14 days can successfully clear gametocytes from blood with disappearance of clinical signs (Eliase and Homans, 1988; Baneth and Weigler, 1997). Generally prognosis is good except guarded prognosis in cases of dogs with high parasitemia.

Hepatozoon americanum species of parasite was discovered in South America on the basis of phylogenetic analysis, geographic distribution, vectors and pathogenesis (Baneth, et al., 2000). $H$. americanum infection also called as American Canine Hepatozoonosis (ACH) which is transmitted by Amblyomma maculatum ticks and reported across southern Gulf States of USA (Gavazza et al., 2003). $\mathrm{ACH}$ attacks skeletal muscles and myocardium which is responsible for clinical syndrome that differs from HCI. It produces more severe infection characterized by fever, lethargy, weight loss, stiffness, paralysis, signs of pain, and mucopurulent ocular discharge. Infection is diagnosed by identifying organism as 'onion skin cysts' in large muscles of infected dogs (Panciera et al., 1999). Researchers at Oklahoma state university have developed indirect ELISA using sporozoites as antigens to diagnose the $\mathrm{ACH}$ infection (Mathew et al., 2001). Compared to HCI, ACH is frustrating to treat. Infection responds poorly to treatment regimen. Management of intense pain is done with non-steroidal anti-inflammatory drugs and supportive care with provision of food and maintaining hydration of dog (Vincent-Johnson et al., 1997). A novel therapeutic approach with Potentiated sulfonamide (Sulfonamide + Trimethoprim) @ 15 $\mathrm{mg} / \mathrm{kg}$ orally every 12 hours for 14 days with $10 \mathrm{mg} / \mathrm{kg}$ of Clindamycin orally every 8 hours for 14 days, Pyrimethamine @ $0.25 \mathrm{mg} / \mathrm{kg} /$ day orally for 14 days followed by Decoquinate @ 10-20 mg/kg orally every 12 hours for continuous 2 years have show promising response in infected dogs but relapses occurs if treatment is discontinued (Macintire et al., 2001).

\section{Ehrlichiosis}

It is a set of syndromes produced by tick transmitted gram negative obligate intracellular Rickettsia of Ehrlichia genus that primarily infects leukocytes (monocytes, macrophages, and granulocytes). Syndromes in canine ehrlichiosis include canine monocytotropic ehrlichiosis, and canine granulocytotropic ehrilichiosis etc. But cross-reactivity and coinfections commonly observed in dogs (Waner et al., 2001). Ehrlichiosis generally presents as non-specific multisystemic disorder with clinical signs of lethargy, depression, weight loss, vomiting, diarrhea and anorexia. Affected dogs may show bleeding tendency due to platelet defects or thrombocytopenia. Other pathologies include uveitis, polymyositis, polyarthritis, and signs of central nervous system affection (Neer $e t$ al., 2002). Hematological abnormalities include nonregenerative anemia and thrombocytopenia while hyperglobulinemia, hypoalbuminemia and low albumin-globulin ratio are the biochemical abnormalities reported in canine Ehrlichiosis (Neer and Harrus, 2006).

Canine Monocytic Ehrlichiosis (CME) : CME is extensively studied ehrlichial disease of dogs caused by infection with Ehrlichia canis and characterized by thrombocytopenia and bleeding tendencies. Three species of E. canis genogroup (I) viz., E. canis, E. chaffeensis, and $E$. ruminantium infect the monocytes of dogs. Geographical distribution of each species varies as so vector tick. Rhipicephalus sanguineus is the vector for E. canis, also experimental transmission with Dermacentor variabilis has been reported 
(Johnson et al., 1998). E. chaffeensis is transmitted by Amblyoma americanum and also by Dermacentor variabilis while no reported evidence about the tick vector of E. ruminantium for transmission in canines is available. Several factors affect the pathogenesis of CME like size of inoculum, immunocompetence of host etc. German shepherd breed of dog is more susceptible to CME with more severe disease and poor prognosis that could be due to depressed cell mediated immunity in these dogs (Neer and Harrus, 2006).

In infected ticks ehrlichae organisms are distributed by hemocytes from the gut to the salivary gland. During feeding, ticks inject salivary secretions containing organisms into feeding sites which causes transmission in dogs. Ticks are able to overwinter and transmit infection for 155 days from infection. Organisms multiply in macrophages of MNS by binary fission and spread throughout body leading to acute, subclinical or chronic syndrome. CME is characterized by development of leucopenia, anemia and thrombocytopenia. Clinically it is a multisystemic disorder characterized by depression, lethargy, weight loss, and anorexia with or without bleeding tendencies. Dogs also exhibit ocular signs (anterior uveitis), neuromuscular signs, and polyarthritis. Concurrent or secondary infections may aggravate the condition of dogs.

CME diagnosed on the basis of combination of clinical signs, hematological deviations, thrombocytopenia, and serological tests. Definitive diagnosis is made on the basis of demonstration of morulae in leukocytes from blood smears or tissue aspirates of lung, spleen and lymph nodes. Serological diagnosis is performed with IFAT and ELISA. A sensitive tool for ehrlichia detection is PCR method which detects DNA of organism. All diagnostics have their pros and cons in diagnosis, but effective and quick test should be utilized for detecting infection that will help in institution of treatment. Effective drugs against CME are tetracyclines, chloramphenicol, imidocarb dipropionate and amicarbalide. There are several controversies regarding effective drug, dose, duration of treatment, efficacy of therapy etc. Doxycycline @ $10 \mathrm{mg} / \mathrm{kg} /$ day orally for minimum 28 days is the standard treatment commonly followed as per American College of Veterinary Internal Medicine (Neer and Harrus, 2006). Oxytetracycline @ $22 \mathrm{mg} / \mathrm{kg}$ orally every 8 hours daily for 28 days or imidocarb dipropionate@5 mg/kg and amicarbalide@ 5-6 $\mathrm{mg} / \mathrm{kg}$ once every 2-3 weeks are the efficacious treatment regimens for CME. Simultaneous short term treatment with Prednisolone @ 2 mg/kg for 2-7 days may be beneficial. Immunosuppressive activity of Prednisolone might have role in to curtail immune mediated mechanisms partially responsible for thrombocytopenia and decreased platelet functions (Frank and Breitschwerdt, 1999; Neer and Harrus, 2006). Supportive therapy in the form of parenteral crystalloid or colloids or blood transfusion according to severity of disease has been indicated (Huxsoll et al., 1970; Neer and Harrus, 2006).

Canine Granulocytic Ehrlichiosis (CGE): CGE is a disease of neutrophils caused by Ehrlichia ewingii and reported only in USA. Organism of CGE is transmitted by several ticks like Ixodes pacificus, Dermacentor variabilis, Rhipicephalus sanguineus, Amblyomma americanum and Ixodes scapularis (Goldman et al., 1998). Disease is characterized by fever, lethargy, anorexia, weight loss, vomiting, diarrhea, transient thrombocytopenia which tend to be severe, and nonregenerative anemia. It also produces acute polyarthritis syndrome as well as neurological disturbances (Maretzki et al., 1994). Clinical and hematological abnormalities of E. ewingii infection are similar to those produced by $E$. canis but appear to be milder than later. Diagnosis of CGE is based on the complete history, clinical findings, hematological findings, demonstration of morulae in granulocytes or synovial fluid, serology and PCR. The recommended protocol for the treatment of CGE is Tetracyclines @ $22 \mathrm{mg} / \mathrm{kg}$ orally every 8 hours for 14-21 days or Doxycycline @ $5-10 \mathrm{mg} / \mathrm{kg}$ orally every $12-24$ hours for 10 days. Other drugs like Minocycline, chloramphenicol can also used by weighing the benefit and adverse effects on host body. There are no evidences suggesting persistence of $E$. ewingii organism after undertaking recommended treatment protocol (Greig et al., 2006).

Hemoprotozoon infections of canines are very dreadful to the health of canines as they affect several body systems including blood and blood forming organs. Studies regarding prevalence of hemoprotozoon diseases in India are partial despite climatic conditions that are frequently adorable for the transmission of vector borne hemoprotozoon diseases in canines (Azaziah et al., 2010). With advent of molecular tools it is now becoming necessary to encrypt the prevalence of these infections in order to take measures for prevention and control of the same.

\section{References}

1. Azaziah, P., Rani, M.A., Irwin, P.J., Gatne, M., Coleman, G.T. and Traub, R.J. (2010). Canine vector borne diseases in India: a review of literature and identification of existing knowledge gaps. Parasites and Vectors, doi: 10.1186/17563305-3-28. 
2. Baneth, G. and Weigler, B. (1997). Retrospective casecontrol study of Hepatozoonosis in dogs in Israel. J. Vet. Int. Med.,11:365-376.

3. Baneth, G., Barta, J.R., Shkap, V., Martin, D.S., Macintire, D.K. and Vincent-Johnson, N. (2000). Genetic and antigenic evidence supports the separation of Hepatozoon canis and Hepatozoon americanum at the species level. J. Clin. Microbiol., 38: 1298-1301.

4. Birkenheuer, A.J., Levy, M.G. and Breitschwerdt, E.B. (2003). Development and evaluation of seminested PCR for detection and differentiation of Babesia gibsoni (Asian genotype) and B. canis DNA in canine blood samples. $J$. Clin.Microbiol., 41(9): 4172-4177.

5. Birkenheuer, A.J., Levy, M.G. and Breitschwerdt, E.B (2004). Efficacy of combined atovaquone and azithromycin for therapy of chronic Babesia gibsoni (Asian genotype) infections in dogs. J. Vet. Intern. Med., 18:494-498.

6. Birkenheuer, A.J., Neel, J., Ruslander, d., Levy, M.G., Breitschwerdt, E.B. (2004). Detection and molecular characterization of novel large Babesia species in dog. Vet. Parasitol., 124: 151-160.

7. Boozer, A.L. and Macintire, D.K. (2003). Canine Babesiosis. Vet Clinics North Am Small Anim Pract., 33: 885-904

8. Camacho-Garcia, A.T. (2006). Piroplasma infection in dogs in northern Spain. Vet. Parasitol., 138(1-2):97-102.

9. Carret, C., Walas, F., Carcy, B., Grande, N., Precigout, E., Moubri, K., Schetters, T. and Gorenflot, A. (1999). Babesia canis, Babesia canis vogeli, Babesia canis rossi Differentiation of three species by restriction fragment length polymorphism analysis on amplified small subuni ribosomal RNA genes. J. Eukaryot. Microbiol., 46: 298-303.

10. Chaudhari, S. (2006). Studies on clinico-therapeutic aspects of babesiosis in dogs. In MVSC Thesis Submitted to Deemed University, Indian Veterinary Research Institute, Izatnagar.

11. Craig, T.M. (2006). Hepatozoonosis, In C.E. GreeneInfectious diseases of the Dog and Cat. $3^{\text {rd }}$ Edn. The W.B. Saunders Co., Philadelphia, Pa.,pp. 698-711.

12. Eliase, E. and Homans, P.A. (1988). Hepatozoon canis infection in dogs: Clinical and hematological findings; treatment. J. Small Anim. Pract., 29: 55-62.

13. Frank, J.R. and Breitschwerdt, E.B. (1999). A retrospective study of Ehrlichiosis in 62 dogs from North Carolina and Virginia. J. Vet. Intern. Med., 13: 194-201.

14. Gallego, L.S. (2008). Whats new about Canine and Feline Babesiosis? Proceedings of the Souhtern European Veterinary Conference (SEVC), Oct. 17-19, Barcelona, Spain.

15. Gavazza, A., Bizzeti, M. and Papini, R. (2003). Observations on dogs found naturally infected with Hepatozoon canis in Italy. Revue Med. Vet., 154 (8-9): 565-571.

16. Goldman, E.E., Breitschwerdt, E.B., Grindem, C.B., Hegarty, B.C., Walls, J.J. and Dumler, J.S. (1998). Granulocytic Ehrlichiosis in dogs from North Carolina and Virginia. J.Vet. Intern. Med., 12: 61-70.

17. Gonen, L., Strauss-Ayali, D., Shkap, V., Vincent-Johnson, N., Macintire, D.K. and Baneth, G. (2004). An enzyme linked immunosorbent assay for antibodies to Hepatozoon canis. Vet. Parasitol., 122 (2): 131-139.

18. Greig, B., Breitschwerdt, E.B. and Armstrong, P.J. (2006) Canine Granulocytotropic Ehrlichiosis. In: Greene, C.E (ed): Infectious Diseases of the Dog and Cat, 3 rd ed. W.B. Saunders Co., Philadelphia, pp.216-232.

19. Gupta, M.P., Nauriyal, D.C., Juyal, P.D., Kalra, I.S., Khahra, S.S. and Mohan, R. (1994). Therapeutic trials of Hepatozoon canis infection in dogs. Ind. Vet. J., 71: 1221

20. Huxsoll, D.L., Hildebrandt, P.K., Nims, R.M. and Walker, J.S. (1970). Tropical canine pancytopenia. J. Am Vet. Med. Assoc., 157: 1627-1632

21. Irwin, P. J. (2009). Canine babesisosis: from molecular taxonomy to control. Parasites and Vectors, 2(1): 1-9.

22. Johnson, E.M., Ewing, S.A., Barker, R.W., Fox, J.C., Crow, D.W. and Kocan, K.M. (1998). Experimental transmission of Ehrlichia canis (Rickettsiales: Ehrlichieae) by Dermacentor variabilis (Acari: Ixodidae). Vet. Parasitol., 74(2-4): 277-288.

23. Juyal, P.D., kalra, I.S. and Singhla, L.D. (1994). Prevalence of hemoprotozooans in domestic animals in Punjab. $6^{4}$ National Congress of Veterinary Parasitology.

24. Kjemtrup, A.M. and Conrad, P.A. (2006). A review of the small canine piroplasms from California: Babesia conradae in the literature. Vet. Parasitol., 138(1-2):112-117.

25. Lakshmanan, B., John, L., Gomathinayagam, S. and Dhinakarraj, G. (2007). Molecular detection of Ehrlichia canis from blood of naturally infected dogs in India. Vet. Archiv., 77: 165-166.

26. Macintire, D.K., Vincent-Johnson, N.A., Kane, C.W., Lindsay, D.S., Blagburn, B.L. and Dillon, A.R. (2001). Treatment of dogs infected with Hepatozoon americanum 53 cases (1989-1998). J. Am Vet. Med. Assoc., 218: 77-82.

27. Maretzki, C.H., Fisher, D.J. and Greene, C.E. (1994) Granulocytic Ehrlichiosis and meningitis in dog. J. Am Vet. Med.Assoc., 205: 1554-1556.

28. Mathew, J.S., Saliki, J.T., Ewing, S.A., Lehenbauer, T.W., Panciera, R.J., Malayer, J.R., Cummings, C.A. and Kocan, A.A. (2001). An indirect enzyme-linked immunosorbent assay for diagnosis of American canine Hepatozoonosis. Vet. Diag. Invest. 13: 17-21.

29. Neer, T.M. and Harrus, S. (2006). Canine Monocytotropic Ehrlichiosis. In: Greene, C.E. (ed): Infectious Diseases of the Dog and Cat, 3 rd ed. W.B. Saunders Co., Philadelphia, pp.203-216.

30. Neer, T.M., Breitschwerdt, E.B., Greene, R.T. and Lappin, M.R. (2002). Consensus statement on ehrlichial disease of small animals from the Infectious Disease Study Group of the ACVIM. J. Vet. Intern. Med., 16:309-315.

31. Panciera, R.J., Ewing, S.A., Mathew, J.S., Lehenbauer, T.W., Cummings, C.A. and Woods, J.P. (1999). Canine Hepatozoonosis: Comparison of lesions and parasites in skeletal muscles of dogs experimentally and naturally infected with Hepatozoon americanum. Vet. Parasitol., 82: 261-272.

32. Pawar, S.D. and Gatne, M.L. (2005). Some hematological and biochemical profiles in canine Hepatozoonosis. J. Vet. Parasitol., 19(2): 171-172.

33. Samaradni, D. Maske, D.K, Kolte, S.W and Shinde, P.N. (2003). Ehrlichiosis in dogs in Nagpur. J. Vet. Parasitol., 17:165-166.

34. Sharma, R.L., Ranga Rao, G.S.C. and Varshney, J.P. (1997). Parasitic diseases of canines- An overview. Int. J. Anim. Sci., 12: 231-244.

35. Sundar, N., Balachandran, C. and Senthivelan, A. (2004): Incidence of Babesia gibsoni infection in dogs in Tamil Nadu. J. Vet. Parasitol., 18(1):79-80.

36. Suzuki, K., Wakabyashi, H., Takahashi, M., Fukushima, K., Yabuki, A. and Endo, Y. (2007). A possible treatment strategy and clinical factors to estimate the treatment response in Babesia gibsoni infection. J. Vet. Med. Sci., 69: 563-568.

37. Taboada, J. and Lobetti, R. (2006). Babesiosis. In: Greene, 
C.E., ed. Infectious Disease of the Dog and Cat. 3rd ed. St Louis, MO: WB Saunders Co; 722-736.

38. Vincent-Johnson, N.A., Macintire, D.K. and Baneth, G. (1997). Canine Hepatozoonosis: Pathophysiology, diagnosis and treatment. Compend. Cont. Ed. Pract. Vet., 19: 51-65.

39. Waner, T., Harrus, S., Jongejan, F., Bark, H., Keysary, A. and Cornelissen, A(2001). Significance of serological testing for ehrlichial diseases in dogs with special emphasis on the diagnosis of canine monocytic ehrlichiosis caused by Ehrlichia canis. Vet. Parasitol., 95:1-15.

40. Wang, C., Ahluwalia, S.K., Li, Y., Dao, D., Poudel, A.,
Chowdhury, E., Boudreaux, M.K. and Kaltenboeck, B (2010). Frequency and therapy monitoring of Babesia spp. infection $b$ high-resolution melting curve quantitative FRET-PCR. Vet. Parasitol., 168 (1-2): 11-18.

41. Yeagley, T.J., et.al. (2009). Detection of Babesia gibsoni and canine small Babesia 'Spanish isolate' in blood samples obtained from dogs confiscated from dog fighting operations. J. Am Vet. Med. Assoc., 235 (5): 535-539.

42. Zahler, M., Schein, E., Rinder, H. and Gothe, R. (1998). Characteristic genotypes discriminate between Babesia canis isolates of differing vector specificity and pathogenicity in dogs. Parasitol. Res., 84: 544-548. 